\title{
EDITORIAL
}

\section{ENTORNOS LABORALES LIBRES DE HUMO DE TABACO: MEJORA DE LA SALUD Y EL BIENESTAR DE LAS PERSONAS EN EL TRABAJO}

Inge van den Borne (1), Tamara Raaijmakers (1), Sibylle Fleitmann (2) y Trudy Prins (2)

(1) Centro Holandés de Fomento de la Salud en el Trabajo, Woerden

(2) Red Europea de Prevención del Tabaquismo, Bruselas

\section{INTRODUCCIÓN}

El establecimiento de lugares de trabajo libres de humo de tabaco constituye un punto importante en la reducción de la mortalidad prematura y la morbilidad evitable entre la población activa.

Según las cifras más recientes de EUROSTAT, en 1999 la Unión Europea contaba con una población activa integrada por 162 millones de ciudadanos. La tasa de empleo era del $72 \%$ entre los hombres y del $53 \%$ entre las mujeres, en la población comprendida entre las edades de 15 y 64 años. En la UE, para los trabajadores a tiempo completo la semana laboral es de 40 horas. Estas personas pasan alrededor de un tercio de sus vidas en el lugar de trabajo. Todos los trabajadores tienen derecho a un ambiente laboral seguro y saludable, que debería garantizarse en la legislación laboral sobre salud y seguridad.

Esta cuestión atañe de manera especial a las mujeres. Mientras la prevalencia del tabaquismo entre la población masculina

Correspondencia:

Sibylle Fleitmann

Red Europea de Prevención del Tabaquismo

144 Chaussée d'Ixelles

1050 Bruselas, Bélgica

Correo electrónico: info@ensp.org tiende paulatinamente a disminuir, entre las mujeres está aumentando, con unas consecuencias devastadoras para su salud. La tasa de empleo femenino está subiendo en toda Europa y se observa, en los últimos 5/6 años, una feminización de los lugares de trabajo. Además, un $80 \%$ de las mujeres trabajan en el sector servicios, muchas en restaurantes y bares. Según las estadísticas, un 73\% de las mujeres que no fuman respira aire contaminado por el humo de tabaco, factor contaminante que además, se ha demostrado particularmente perjudicial para las mujeres embarazadas.

Sólo se puede elaborar una política realista sobre lugares de trabajo libres de humo de tabaco si se involucra a todos los interesados en el proceso. Se ha de elevar el nivel de concienciación, difundir los datos científicos y servirse de argumentos pragmáticos de relevancia en el contexto cotidiano de la realidad laboral.

Este informe de la situación en Europa representa el punto culminante de un estudio reciente y de las conclusiones de una Conferencia Europea celebrada en Berlín los días 10 y 11 de mayo de 2001. En este marco, el informe se fundamenta en la investigación académica disponible, junto con la experiencia práctica para elaborar argumentos sólidos y contundentes que apuntan a la necesi- 
dad de promover cambios significativos en el ámbito laboral. Nuestro deseo es que:

- sirva como un documento de alcance global y como fuente de referencia para difundir argumentos a favor del establecimiento de lugares de trabajo libres del humo de tabaco.

- impulse un debate entre los defensores de la salud pública, las empresas y los responsables políticos.

- influya y genere políticas/normativas de ámbito nacional y europeo.

Y se ha preparado con este fin.

\section{El cúmulo de evidencia}

Desde 1986, en un total de 14 informes bien documentados y elaborados por instituciones médicas y medio-ambientales, así como agencias oficiales en Europa, Norteamérica y Australia, se ha establecido que el consumo involuntario del tabaco constituye un riesgo para la salud pública. Algunas de estas instituciones, como por ejemplo el National Institute on Occupational Safety and Health (Instituto Nacional de la Seguridad y Salud en el Trabajo - NIOSH) de los EEUU, ha clasificado el aire contaminado por humo de tabaco (ACHT) como factor cancerígeno reconocido.

El ACHT contiene muchos compuestos químicos, más de 40 de los cuales son agentes cancerígenos en los animales y en los seres humanos. El ACHT también lleva compuestos químicos que irritan los ojos y las vías respiratorias altas. La 'corriente secundaria' del humo de tabaco que emite un cigarrillo prendido contiene concentraciones más altas de dichos compuestos que la 'corriente principal' que el fumador inspira directamente.

Los niños son las víctimas más vulnerables del consumo involuntario del tabaco.
Ante la exposición al ACHT, aumenta en ellos el riesgo de que sufran el síndrome de muerte súbita infantil (SIDS), infecciones de las vías respiratorias altas, asma bronquial e infecciones del oído medio. Entre los adultos que no fuman, el consumo involuntario de tabaco aumenta el riesgo de cáncer de pulmón y de las enfermedades coronarias y cerebrovasculares.

En Europa, al igual que en otras zonas del mundo, paulatinamente se han ido reconociendo los efectos a largo plazo de la exposición al ACHT en el medio laboral. Los quince Estados Miembros de la UE ya cuentan con alguna normativa que regula el consumo de tabaco en los lugares públicos, pero en muy pocos países esta legislación hace referencia explícita al medio laboral. La mayor parte de leyes actualmente vigentes contemplan las restricciones del consumo de tabaco en los centros sanitarios, edificios públicos y transporte público, que por otra parte también son lugares de trabajo.

\section{¿Qué hacer?}

En el ámbito europeo, hay dos directivas que tratan del consumo de tabaco en el lugar de trabajo, pero de forma muy limitada. Una de ellas, una directiva del año 1983 (83/477) estipula que no se pueda fumar en las zonas en las que los trabajadores están expuestos al polvo de amianto. La otra, de 1989 (89/654), se refiere a la calidad del aire en los espacios de trabajo cerrados. En concreto, exige que se tomen las medidas adecuadas para la protección de los no fumadores en los lugares reservados para el descanso de los trabajadores.

En ocho de los quince Estados Miembros de la UE se han producido litigios en defensa de los derechos de las personas que no fuman: Alemania, Dinamarca, Francia, Irlanda, Italia, Países Bajos, el Reino Unido y Suecia. En dichas causas a menudo se apela a la normativa vigente que obliga a las 
empresas a proporcionar un entorno de trabajo seguro, argumentando que los empleados que se ven obligados a trabajar en un ambiente lleno de humo se exponen a un riesgo laboral.

Se han implantado -con éxito- políticas para el control del tabaquismo en lugares de trabajo de diferentes tipos y tamaños en muchos países de Europa, Norteamérica y otras zonas del mundo. Tras más de 20 años de experiencia en la redacción y puesta en práctica de dichas políticas se han ido perfilando con mucha nitidez los principios clave de una política eficaz. Estos principios básicos incluyen:

- Enfatizar que las molestias las provoca el humo de tabaco, no las personas que fuman.

- Centrarse en los derechos de los individuos a disfrutar un ambiente saludable y seguro.

- Conseguir el compromiso y el apoyo de la dirección.

- Brindar a los empleados la oportunidad de participar en la elaboración y puesta en marcha de la política.

- Orientar a la dirección de la empresa cómo difundir y hacer cumplir las normas que resultan de la política adoptada.

- Informar a todo el personal sobre los riesgos del ACHT en el ámbito laboral.

- Asegurarse de que las restricciones son iguales y que se aplican con el mismo rigor en todos los niveles de la organización.

- Prever plazos suficientemente amplios para anunciar la política, realizar la planificación y poner en práctica el nuevo sistema.

- Brindar a los empleados y a sus familias ayuda para abandonar el consumo de tabaco antes y después del cambio de política.

- Realizar un seguimiento continuado de la política adoptada.

\section{Perspectivas}

Las políticas sobre el tabaquismo en el lugar de trabajo benefician tanto a los fumadores como a los no fumadores. La investigación ha demostrado que gracias a las políticas sobre tabaquismo en el lugar de trabajo se reduce el consumo diario de cigarrillos entre las personas que fuman. La probabilidad de dejar de fumar es más alta entre los fumadores que trabajan en lugares de trabajo libres de humo de tabaco que entre los que trabajan en empresas que carecen de una política al respecto.

El absentismo es más alto entre los fumadores que los no fumadores. Las bajas entre las personas que fuman se deben no sólo a enfermedades graves, como puede ser una enfermedad coronaria o respiratoria, sino también al hecho de que los empleados fumadores son más susceptibles a la tos, los resfriados y la gripe.

Los costes del absentismo laboral no se limitan a la prestación por enfermedad sino que incluyen además los costes derivados de las pérdidas de eficacia y productividad atribuibles a la falta de personal o del empleo de personal temporal sin experiencia.

Por otra parte, el consumo de tabaco en el lugar de trabajo puede acarrear costes adicionales a la empresa, que incluyen conceptos como el aumento de las primas de seguros o los daños al mobiliario, la decoración y los equipos provocados por el ACHT. Una política sobre el consumo del tabaco en el lugar de trabajo también puede mejorar la imagen de la empresa y reducir los conflictos entre los empleados fumadores y no fumadores.

Sin duda, la elaboración y puesta en práctica de una política sobre tabaquismo en el 
lugar de trabajo implican algunos costes, tales como el gasto de construir y ventilar las salas de fumadores, la compra de programas para dejar de fumar, etc. No obstante, los análisis de las ventajas a largo plazo de dichas políticas demuestran que se trata de inversiones económicas rentables que redundan en el ahorro a largo plazo, proporcionando, además, beneficios menos tangibles como son la elevación de la moral de la plantilla y la mejora de la imagen corporativa.

\section{AGRADECIMIENTOS}

Tanto la Conferencia como el informe se han realizado en el marco de un proyecto más amplio de la Red Europea de Prevención del Tabaquismo, que ha sido posible gracias a la financiación del programa Europa Contra el Cáncer de la Comisión Europea. La traducción y revisión técnica de los documentos las ha realizado el Centro de Estudios sobre Promoción de la Salud de Madrid. Damos las gracias a las siguientes personas por la revisión crítica que han realizado de los informes incluidos en esta publicación: Grieto Zeeman (Defacto, Países Bajos), Friedrich J. Wiebel (National Research Center for Environment and Health, Alemania), Patti White (Health Eduction Authority, Inglaterra). 